\title{
G

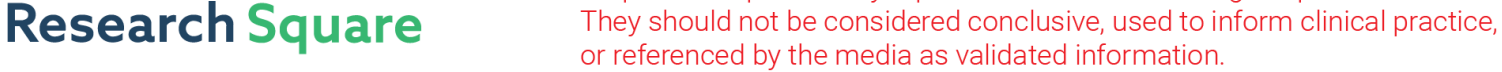 \\ Clinical Characteristics of Choledochal Cysts With Intrahepatic Bile Duct Dilatations
}

\section{Shuhao Zhang}

The Children's Hospital, Zhejiang University School of Medicine, National Clinical Research Center For Child Health

\section{Duote Cai}

The Children's Hospital, Zhejiang University School of Medicine, National Clinical Research Center For Child Health

\section{Yuebin Zhang}

The Children's Hospital, Zhejiang University School of Medicine, National Clinical Research Center For Child Health

\section{Ken Chen}

The Children's Hospital, Zhejiang University School of Medicine, National Clinical Research Center For Child Health

\section{Yi Jin}

The Children's Hospital, Zhejiang University School of Medicine, National Clinical Research Center For Child Health

\section{Wenjuan Luo}

The Children's Hospital, Zhejiang University School of Medicine, National Clinical Research Center For Child Health

\section{Zongwei Huang}

The Children's Hospital, Zhejiang University School of Medicine, National Clinical Research Center For Child Health

\section{Di Hu}

The Children's Hospital, Zhejiang University School of Medicine, National Clinical Research Center For Child Health

\section{Zhigang Gao (D6519040@zju.edu.cn )}

The Children's Hospital, Zhejiang University School of Medicine, National Clinical Research Center For Child Health

\section{Research Article}

Keywords: choledochal cyst, intrahepatic bile duct, extrahepatic bile duct, dilatation

Posted Date: October 18th, 2021 
DOI: https://doi.org/10.21203/rs.3.rs-955070/v1

License: (c) (1) This work is licensed under a Creative Commons Attribution 4.0 International License. Read Full License 


\section{Abstract}

Background: Whether the dilated intrahepatic bile duct (IHBD) has any effect on prognosis of choledochal cyst (CC) remains controversy. In the study, we aim to summarize the clinical characteristics and prognosis of those patients.

Methods: A total of 192 children (47 males, 145 females) diagnosed with CC were identified in our hospital, including 127 without IHBD dilatation (group A) and 65 with IHBD dilatation (group B). A retrospective analysis was performed to explore the clinical characteristics of group $B$ based on clinical indices, symptoms, and complications.

Results: Compared with group $A$, incidences of jaundice and fever were significantly increased in group $B$ $(p=0.01$ and 0.033 , respectively). Preoperative total bilirubin (TB), direct bilirubin (DB), and indirect bilirubin (IDB) were also higher in group $B$ than in group $A(p=0.0052,0.0005$, and 0.0136 , respectively), as were preoperative levels of alanine aminotransferase (ALT), aspartate aminotransferase (AST), gamma-glutamyl transferase (GGT), and total bile acid (TBA) $(p=0.0057,0.0250,0.0002$, and 0.0242 , respectively). Early postoperative GGT levels in group $B$ remained abnormal and were significantly higher than in group $A(p=0.0003)$. The risk of liver fibrosis or cirrhosis was significantly increased for group $B$ compared with group $A(p=0.012)$ and also occurred earlier in group $B(p<0.001)$, among them, dilated IHBDs recovered to normal in $89.23 \%(7 / 65)$ of patients. Meanwhile, the incidence of postoperative pancreatitis increased significantly in group $B$ as well $(p=0.003)$.

Conclusion: CC with IHBD dilatation was associated with significantly increased preoperative bilirubin levels, abnormal liver function, and higher incidence of liver fibrosis or cirrhosis early in the disease course. Timely radical surgery is recommended for such patients and postoperative extinction of the IHBD dilatation occurs early in most of those patients.

\section{Background}

Choledochal cysts (CCs) are cystic or fusiform dilations of the common bile duct. As advanced imaging techniques facilitate improved CC diagnostics, its incidence has increased, particularly in neonates due to a distinct increase in prenatally diagnosed CC[1]. The clinical features of CC differ according to age. Neonates and young infants usually present with an abdominal mass, jaundice, or acholic stools, depending on the degree of obstruction. To date, $\mathrm{CC}$ is primarily grouped according to the Todani classification that was proposed in 1977, in which Todani type IV-a cysts are accompanied by IHBD dilation. The incidence of IHBD dilatation accounts for $30-40 \%$ of all CC cases[2]. After properly dealing with the EHBDs, spontaneous extinction of the IHBD dilatation occurs early in some cases; however, some IHBD dilatation is persistent, and whether this residual dilatation has any effect on the long-term prognosis of children with CC is unclear.

In this study, we reviewed our institution's records from October 2016 to December 2019 for cases of CCs, which revealed that CC with IHBD dilatation had conspicuous pre- and postoperative clinical 
characteristics compared with CC cases without IHBD dilatation. Thus, we summarized the clinical characteristics and outcomes of CC with IHBD dilatation based on the clinical symptoms, laboratory indices, and liver biopsies to establish guidelines for the future clinical management of this disease.

\section{Methods}

\subsection{Inclusion criteria}

The inclusion criteria for group B included: 1) a preoperative abdominal scan [B ultrasound, computed tomography (CT), or magnetic resonance cholangiopancreatography (MRCP)] showed moderately-toseverely dilatated IHBD[3]; 2) dilatated EHBDs were identified during surgery; 3) postoperative pathological confirmation of CC; and 4) well-documented clinical data were available. The remaining CC patients were included in group $A$.

\subsection{Clinical data}

In total, data from $192 \mathrm{CC}$ patients (47 males and 145 females) who were treated at the Department of General Surgery, Children's Hospital of Zhejiang University School of Medicine between October 2016 and December 2019 were collected. Group A included 127 CC patients (31 males and 96 females) without IHBD dilatation. The average operative age of group A was 24.00 months [interquartile range (IQR): 5.3059.00 months], the average postoperative hospital stay was $13.00 \mathrm{~d}$ (range: $11.00-15.00 \mathrm{~d}$ ), and the average cyst diameter was $22.00 \mathrm{~mm}$ (IQR: $14.50-35.00 \mathrm{~mm}$ ). Group B included $65 \mathrm{CC}$ patients (16 males and 49 females) with IHBD dilatation. The average operative age of group B was 23.00 months (IQR: $3.35-42.50$ months), the average postoperative hospital stay was $14.00 \mathrm{~d}$ (IQR: $11.00-16.00 \mathrm{~d}$ ), and the average cyst diameter was $27.50 \mathrm{~mm}$ (IQR: 12.13-41.00 mm). The main preoperative clinical symptoms (fever, abdominal pain, jaundice, and vomiting) and pre- and postoperative clinical indices are provided in Table 1. 
Table 1

Clinical characteristics of choledochal cysts with or without intrahepatic bile duct dilatation

\begin{tabular}{|c|c|c|c|}
\hline \multicolumn{4}{|l|}{ Preoperative } \\
\hline & Group A $(n=127)$ & Group B (n=65) & $p$ value \\
\hline Sex (F:M) & 96: 31 & 49: 16 & 0.975 \\
\hline Age (Month) & $24.00(5.30-59.00)$ & $23.00(3.35-42.50)$ & 0.2843 \\
\hline Postoperative hospital stay(Days) & $13.00(11.00-15.00)$ & $14.00(11.00-16.00)$ & 0.2283 \\
\hline Cyst diameter (mm) & $22.00(14.50-35.00)$ & $27.50(12.13-41.00)$ & 0.2352 \\
\hline PT (second) & $11.70(11.00-12.40)$ & $11.70(10.70-13.00)$ & 0.8627 \\
\hline APTT (second) & $29.30(26.70-32.60)$ & $28.80(26.50-32.00)$ & 0.5850 \\
\hline Total bilirubin ( $\mu \mathrm{mol} / \mathrm{L})$ & $10.90(6.40-33.90)$ & $29.55(8.95-79.58)$ & 0.0052 \\
\hline Direct bilirubin ( $\mu \mathrm{mol} / \mathrm{L})$ & $2.80(1.40-11.30)$ & $11.35(2.05-39.50)$ & 0.0005 \\
\hline Indirect bilirubin ( $\mu \mathrm{mol} / \mathrm{L})$ & $8.40(5.10-18.90)$ & $14.95(7.35-43.93)$ & 0.0136 \\
\hline $\mathrm{ALT}(\mathrm{U} / \mathrm{L})$ & $32.00(15.00-85.00)$ & $70.50(20.50-235.00)$ & 0.0057 \\
\hline AST (U/L) & $47.00(34.00-104.00)$ & $67.00(39.25-191.25)$ & 0.025 \\
\hline GGT (U/L) & $79.00(15.00-270.00)$ & $363.00(53.00-614.00)$ & 0.0002 \\
\hline Total bile acid $(\mu \mathrm{mol} / \mathrm{L})$ & $8.20(3.90-16.30)$ & $12.10(3.60-87.30)$ & 0.0242 \\
\hline \multicolumn{4}{|l|}{ Postoperative (at fifth day) } \\
\hline Total bilirubin $(\mu \mathrm{mol} / \mathrm{L})$ & $10.35(7.20-17.83)$ & $16.75(6.70-38.10)$ & 0.0808 \\
\hline Direct bilirubin $(\mu \mathrm{mol} / \mathrm{L})$ & $2.20(1.50-4.78)$ & $3.75(1.63-13.78)$ & 0.0270 \\
\hline Indirect bilirubin ( $\mu \mathrm{mol} / \mathrm{L})$ & 7.90 (5.50-12.08) & $12.85(4.93-23.88)$ & 0.1173 \\
\hline $\operatorname{ALT}(\mathrm{U} / \mathrm{L})$ & $24.00(16.25-40.00)$ & $30.50(21.00-47.50)$ & 0.0212 \\
\hline AST (U/L) & $33.00(26.00-46.00)$ & $37.00(29.25-52.00)$ & 0.0775 \\
\hline GGT (U/L) & $49.50(17.00-168.25)$ & $151.50(49.25-287.75)$ & 0.0003 \\
\hline Total bile acid ( $\mu \mathrm{mol} / \mathrm{L})$ & $6.20(3.13-10.90)$ & $5.80(2.75-10.65)$ & 0.6727 \\
\hline
\end{tabular}

1.3 Diagnosis and treatment

Group A included 31 patients who were diagnosed by B ultrasound, 90 patients diagnosed by MRCP, and six patients diagnosed by $\mathrm{CT}$, among which, 28 patients were detected during prenatal screening. Group $\mathrm{B}$ included 16 patients who were diagnosed by B ultrasound, 46 patients diagnosed by MRCP, and three patients diagnosed by CT, among which, 15 patients were detected during prenatal screening. 
All patients received excision of dilated EHBDs and hepaticojejunostomy, which were performed by three experienced surgeons. Patients received postoperative imipenem-cilastatin sodium as an antimicrobial treatment for 3-to-5 d, then the antibiotic was changed to cephalosporin if white blood count (WBC) and C-reactive protein (CRP) levels decreased and the patient had recovered well. The gastrointestinal decompression tube could be removed $5 \mathrm{~d}$ postoperatively if the patients felt well (without nausea, vomiting, or abdominal distension), then liquid and semi-liquid diets could be gradually introduced. The abdominal drainage tube could be removed when the postoperative daily drainage volume less than 15$20 \mathrm{~mL}$ and the seroperitoneum less than $2 \mathrm{~cm}$.

\subsection{Statistical analysis}

Data are presented as the median \pm IQR. All statistical analyses were performed with SPSS18.0 (SPSS Inc., Chicago, IL, USA) and GraphPad Prism 6 (GraphPad Software, Inc., San Diego, CA, USA) software packages. The independent samples $t$-test was used to compare samples. Comparisons of liver fibrosis and cirrhosis were conducted by the chi-square test. Survival curves were drawn using Kaplan-Meier univariate estimates. P-values $<0.05$ were considered statistically significant.

\section{Results}

\subsection{Preoperative clinical data}

In total, $192 \mathrm{CC}$ patients (47 males and 145 females) were included in this study, among whom 127 (31 males and 96 females) were without IHBD dilatation (group A), and 65 (16 males and 49 females) had IHBD dilatation (group B). The proportions of symptomatic patients were $62.99 \%$ in group A and $75.38 \%$ in group B. In both groups, abdominal pain was the most common symptom (47.24\% in group A and $49.23 \%$ in group B), followed by vomiting, jaundice, and fever. The incidence of jaundice and fever in group $B$ were significantly higher than in group $A(p=0.01$ and $p=0.033$, respectively). (Table 2 )

Table 2

Incidence of preoperative symptoms

\begin{tabular}{|llll|}
\hline & Group A $(\mathbf{n}=127)$ & Group B $(\mathbf{n}=65)$ & p value \\
\hline Fever & $3(2.36 \%)$ & $7(10.77 \%)$ & 0.033 \\
\hline Abdominal pain & $60(47.24 \%)$ & $32(49.23 \%)$ & $p>0.05$ \\
\hline Jaundice & $19(14.96 \%)$ & $20(30.77 \%)$ & 0.01 \\
Vomit & $42(33.07 \%)$ & $26(40.00 \%)$ & $p>0.05$ \\
\hline Pancreatitis & $18(14.17 \%)$ & $12(18.46 \%)$ & $p>0.05$ \\
\hline
\end{tabular}

The diameters of the cysts were measured by B ultrasound, CT, or MRCP and were not significantly different between the two groups [22.00 mm (IQR: 14.50-35.00 mm) vs. $27.50 \mathrm{~mm}$ (IQR: 12.13-41.00 $\mathrm{mm}$ )]. Preoperative PT and APTT were also not significantly different between the two groups [PT: 11.70 
(IQR: 11.00-12.40) vs. 11.70 (IQR: 10.70-13.00); APTT: 29.30 (IQR: 26.70-32.60) vs. 28.80 (IQR: 26.50$32.00)]$. The incidence of jaundice in group $B$ was significantly higher than in group $A(p=0.01)$, and preoperative total bilirubin, direct bilirubin, and indirect bilirubin levels were also higher in group $B$ $(p=0.0052, p=0.0005$, and $p=0.0136$, respectively). Compared with group $A$, preoperative liver function (ALT and AST) was significantly increased in group $B(p=0.025$ and $p=0.0057$, respectively). Finally, preoperative GGT levels were also significantly higher in group B compared with group A [363.00 (IQR: 53.00-614.00) vs. 79.00 (IQR: 15.00-270.00), p=0.0242]. (Table 1)

\subsection{Postoperative clinical data}

Following surgery, the average levels of total bilirubin, direct bilirubin, indirect bilirubin, ALT, AST, and GGT recovered to normal at fifth day in group $A$; these same indices also recovered to normal at fifth day in group B, except GGT, which remained significantly increased [151.50 (IQR: (49.25-287.75) vs. 49.50 (IQR: 17.00-168.25), $p=0.0003$ ] (Table 1). Further analysis of liver biopsies from the two groups showed that the proportion of liver fibrosis and cirrhosis was significantly increased in group B compared with group $A$ $(p=0.012$; Table 3$)$; furthermore, there was also an increased incidence of postoperative pancreatitis in group $B(p=0.003$; Table 3). Finally, Kaplan-Meier analysis indicated that liver fibrosis and cirrhosis occurred earlier in group $B$ than in group $A(p<0.001$; Figure 1$)$.

Table 3

Postoperative complications

\begin{tabular}{|llll|}
\hline & Group A (n=127) & Group B (n=65) & p value \\
\hline Liver fibrosis or cirrhosis & 18 & 19 & 0.012 \\
\hline Bile leakage & 7 & 1 & $p>0.05$ \\
\hline Anastomotic stenosis & 9 & 1 & $p>0.05$ \\
\hline Pancreatitis & 2 & 8 & 0.003 \\
\hline Ileus & 5 & 3 & $p>0.05$ \\
\hline Pancreatic pseudocyst & 0 & 1 & $p>0.05$ \\
\hline Cholangitis & 1 & 0 & $p>0.05$ \\
\hline Septic shock & 1 & 0 & $p>0.05$ \\
\hline
\end{tabular}

\subsection{Follow-up data}

The average follow-up time were 13.24 (2.45-32.30) and 12.35 (1.63-29.00) months in group A and group $B$, respectively. Dilated IHBDs recovered to normal after surgery in $89.23 \%(58 / 65)$ of patients in group $B$. The average follow-up time of these 7 patients with remained IHBDs dilatation was 6.70 (2.70-17.00) months. 


\section{Discussion}

Type IV-a CC has been reported more and more in recent years, and accounts for up to $29 \%$ of some series[4]. In our series, Type IV-a CC accounts for $33.85 \%$ of all the patients. Most of the dilated IHBDs could recover to normal after removing the obstruction by excision of dilated EHBDs and hepaticojejunostomy; while for some type IV-a CC cases, IHBD dilatation will persist postoperatively [5]. Here, we have shown that CC patients with IHBD dilatation had conspicuous clinical symptoms and needed aggressive intervention.

Patients with initial manifestation of CC usually have nonspecific symptoms, and the classic triad (abdominal pain, jaundice, and abdominal mass) has proven to be rare[2]. In this study, the predominant symptoms were abdominal pain and vomiting, followed by jaundice and fever. And the incidence of the latter two symptoms was much higher in patients with IHBDs dilatation. It is probable that the increased pressure in the biliary tract results from biliary obstruction and poor bile drainage were more prone to cause recurrent fever and jaundice[6, 7]. The significantly elevated pre- and postoperative GGT levels in group B which was related to the biliary obstruction $[8,9]$ further verified the increased biliary pressure. Notably, previous studies also had confirmed a significant stepwise increased pressure between two common types (type $1<$ type 4$)[5,10]$.

Actually, it has been shown that early excision of the extrahepatic portion of the cyst for type IV-a CC without removing the cystic dilation of IHBD provides satisfied prognosis[11, 12]. And previous studies had reported that the intrahepatic portion of the cyst diminished or disappeared after surgery, and that preoperative IHBD dilatation was not significantly associated with prognosis[12, 13]. In our study, type IVa CC patients with early surgery also experienced satisfied outcome and normal liver function even IHBDs dilatation persisted (Figure 2 and Table 4). However, some studies reported that the incidence of malignancy was higher in patients who underwent Roux-en-Y hepaticojejunostomy without radical resection of cysts compared with patients who did not undergo any surgery[14], and the dilated bile ducts might be vulnerable to bile infection, resulting stone formation[15]. And such case that intrahepatic cholangiocarcinoma (IHCC) arising many years after excision of a type IV-a congenital choledochal cyst also have occasionally been reported[16, 17]. According to previous research, the incidence of malignancy before the age of 18 was 0.42 versus $11.4 \%$ in adults[18]. The average follow-up time of our cohort already exceed one year and almost all patients in our study (190/192) except two patients with liver cirrhosis experienced early satisfactory outcomes after complete resection of dilated EHBDs without removing dilated IHBDs. However, those patients with persistent IHBDs dilatation needs longer-term follow-up to confirm whether the remained intrahepatic cysts will influence their symptom-free survival. 
Table 4

The postoperative clinical indices of the same patient in Fig. 2

\begin{tabular}{|lllllll|}
\hline Date & $\begin{array}{l}\text { Total } \\
\text { bilirubin } \\
(\mu \mathrm{mol} / \mathrm{L})\end{array}$ & $\begin{array}{l}\text { Direct bilirubin } \\
(\mu \mathrm{mol} / \mathrm{L})\end{array}$ & $\begin{array}{l}\text { Indirect bilirubin } \\
(\mu \mathrm{mol} / \mathrm{L})\end{array}$ & $\begin{array}{l}\text { ALT } \\
(\mathrm{U} / \mathrm{L})\end{array}$ & $\begin{array}{l}\text { AST } \\
(\mathrm{U} / \mathrm{L})\end{array}$ & $\begin{array}{l}\text { GGT } \\
(\mathrm{U} / \mathrm{L})\end{array}$ \\
\hline 2017.07 .08 & 6.2 & 1.0 & 5.2 & 14 & 46 & 9 \\
\hline 2017.11 .03 & 4.8 & 1.0 & 3.8 & 14 & 45 & 9 \\
\hline 2018.08 .08 & 4.4 & 0.9 & 3.5 & 13 & 35 & 10 \\
\hline 2019.02 .02 & 5.1 & 0.9 & 4.2 & 14 & 40 & 10 \\
\hline 2019.08 .03 & 4.9 & 1.1 & 3.8 & 13 & 38 & 10 \\
\hline 2020.05 .01 & 6.8 & 1.5 & 5.3 & 18 & 39 & 10 \\
\hline
\end{tabular}

Although postoperative hepatic biochemical indices restored to normal early in our cohort, the incidences of liver fibrosis and cirrhosis were significantly increased in group B as well as the incidence of postoperative pancreatitis. Two patients with IHBD dilatation even developed postoperative liver cirrhosis at 8.2 and 9.8 months, respectively. Our Kaplan-Meier analysis used liver fibrosis as a preliminary biomarker for patient assessments[19] and indicated that liver fibrosis or cirrhosis appeared also significantly earlier in type IV-a CC. In a word, CC patients with IHBD dilatation should receive timely surgery as more recent studies have supported the idea that liver fibrosis is a reversible process if the injury-causing stimulus is withdrawn, while liver cirrhosis is not[20,21].

\section{Conclusions}

In conclusion, CC patients with IHBD dilatation had relatively conspicuous preoperative symptoms, highly elevated bilirubin, and abnormal liver function, and they were more prone to develop liver fibrosis or cirrhosis in early stages. Therefore, timely radical surgery is recommended once surgical contraindications are excluded and meticulous follow-up is needed for CC patients with IHBD dilatation. Mostly, patients with IHBDs dilatation experiences satisfactory outcomes and IHBDs dilatation diminish spontaneously in most cases.

\section{Abbreviations}

choledochal cyst (CC), intrahepatic bile duct (IHBD), extrahepatic bile duct (EHBD) $\rrbracket$

total bilirubin (TB), direct bilirubin (DB), indirect bilirubin (IDB), alanine

aminotransferase (ALT), aspartate aminotransferase (AST), gamma-glutamyl

transferase (GGT), interquartile range (IQR). 


\section{Declarations}

Ethics approval and consent to participate

This study was carried out in accordance with the recommendations of the Ethics Committee of The Children's Hospital『Zhejiang University School of Medicine [2020-IRB-055] with written informed consent in accordance with Declaration of Helsinki. The protocol was approved by the Ethics Committee of The Children's Hospital, Zhejiang University School of Medicine and informed consent was obtained all from their parents.

Consent for publication

Not applicable.

Author's contribution

SHZ, DTC, ZGG are expected to have made substantial contributions to the onception and design of the work. SHZ collected the data. SHZ, DTC, YBZ, KC, WJL, YJ and ZGG interpreted the data. SHZ and ZGG drafted the manuscript. DTC, YBZ, KC, WJL, ZWH, DH and ZGG revised the manuscript. All authors read and approved the final draft.

Funding

This work was supported by the Clinical Medical Research of Minimally Invasive Diagnosis and Treatment of Abdominal Organs in Zhejiang Province [grant number: 01492-02].

Availability of data and materials

The datasets used and/or analysed during the current study are available from the corresponding author on reasonable request.

Competing interests

All authors have no conflicts of interest to disclose regarding this study.

Acknowledgement

We thank International Science Editing ( http://www.internationalscienceediting.com ) for editing this manuscript.

\section{References}

1. Matsumoto M, Urushihara N, Fukumoto K, Yamoto M, Miyake H, Nakajima H: Laparoscopic management for prenatally diagnosed choledochal cysts. SURG TODAY 2016, 46(12):1-5. 
2. Koshinaga T, Inoue M, Ohashi K, Sugito K, Ikeda T, Hagiwara N, Tomita R: Persistent biliary dilatation and stenosis in postoperative congenital choledochal cyst.J HEPATO-BIL-PAN SCI 2011, 18(1):47-52.

3. Congo K, Lopes MF, Oliveira PH, Matos H, Reis A: Outcomes of choledochal cysts with or without intrahepatic involvement in children after extrahepatic cyst excision and Roux-en-Y hepaticojejunostomy. ANN HEPATOL 2012, 11(4):536.

4. Saluja SS, Nayeem M, Sharma BC, Bora G, Mishra PK: Management of choledochal cysts and their complications.AM SURGEON 2012, 78(3):284.

5. Hill R, Parsons C, Farrant P, Sellars M, Davenport M: Intrahepatic duct dilatation in type 4 choledochal malformation: pressure-related, postoperative resolution. J PEDIATR SURG 2011, 46(2):299-303.

6. Fujishiro J, Masumoto K, Urita Y, Shinkai T, Gotoh C: Pancreatic complications in pediatric choledochal cysts. J PEDIATR SURG 2013, 48(9):1897-1902.

7. Kaneko K, Ono Y, Tainaka T, Sumida W, Ando H: Fatty acid calcium stones in patients with pancreaticobiliary maljunction/choledochal cyst as another cause of obstructive symptoms besides protein plugs. J PEDIATR SURG 2008, 43(3):564-567.

8. Lieberman MW, Barrios R, Carter BZ, Habib GM, Wan DF: gamma-Glutamyl transpeptidase. What does the organization and expression of a multipromoter gene tell us about its functions? AM J PATHOL 1995, 147(5):1175-1185.

9. Cabrera-Abreu JC, Green A: Gamma-glutamyltransferase: value of its measurement in paediatrics.ANN CLIN BIOCHEM 2002, 39(1):22.

10. Kronfli R, Davenport M: Insights into the pathophysiology and classification of type 4 choledochal malformation. J PEDIATR SURG 2020, 55(12):2642-2646.

11. Zheng X, Gu W, Xia H, Huang X, Liang B, Yang T, Yang S, Zeng J, Dong J: Surgical treatment of type IVA choledochal cyst in a single institution: Children vs. adults. J PEDIATR SURG 2013, 48(10):2061-2066.

12. Ono S, Fumino S, Shimadera S, Iwai N: Long-term outcomes after hepaticojejunostomy for choledochal cyst: a 10- to 27-year follow-up.J PEDIATR SURG 2010, 45(2):376-378.

13. Ohi R, Koike N, Matsumoto Y, Ohkohchi N, Kasai M: Changes of intrahepatic bile duct dilatation after surgery for congenital dilatation of the bile duct. J PEDIATR SURG 1985, 20(2):138-142.

14. Ziegler KM, Zyromski NJ: Choledochoceles: Are They Choledochal Cysts?Advances in Surgery 2011, 45(1):211-224.

15. Uno K, Tsuchida Y, Kawarasaki H: Development of intrahepatic cholelithiasis long after primary excision of choledochal cysts.J Am Coll Surg 1996, 97(8):583-588. 
16. Mizuguchi $Y$, Nakamura $Y$, Uchida E: Subsequent biliary cancer originating from remnant intrapancreatic bile ducts after cyst excision: a literature review. SURG TODAY 2016, 47(6):1-8.

17. Shimamura K, Kurosaki I, Sato D, Takano K, Yokoyama N, Sato Y, Hatakeyama K, Nakadaira K, Yagi M: Intrahepatic cholangiocarcinoma arising $\mathbf{3 4}$ years after excision of a type IV-A congenital choledochal cyst: report of a case.SURG TODAY 2009, 39(3):247.

18. Sastry AV, Abbadessa B, Wayne MG, Steele JG, Cooperman AM: What is the Incidence of Biliary Carcinoma in Choledochal Cysts, When Do They Develop, and How Should it Affect Management? WORLD J SURG 2015, 39(2):487-492.

19. Dong JH, Yang SZ, Xia HT, Duan WD, Ji WB, Gu WQ, Liang B, Huang ZQ: Aggressive hepatectomy for the curative treatment of bilobar involvement of type IV-A bile duct cyst.ANN SURG 2013, 258(1):122-128.

20. Atta HM: Reversibility and heritability of liver fibrosis: Implications for research and therapy.WORLD J GASTROENTERO 2015, 21(17):5138-5148.

21. Ramachandran P, Iredale JP: Reversibility of liver fibrosis - ScienceDirect. ANN HEPATOL 2009, 8(4):283-291.

Figures 


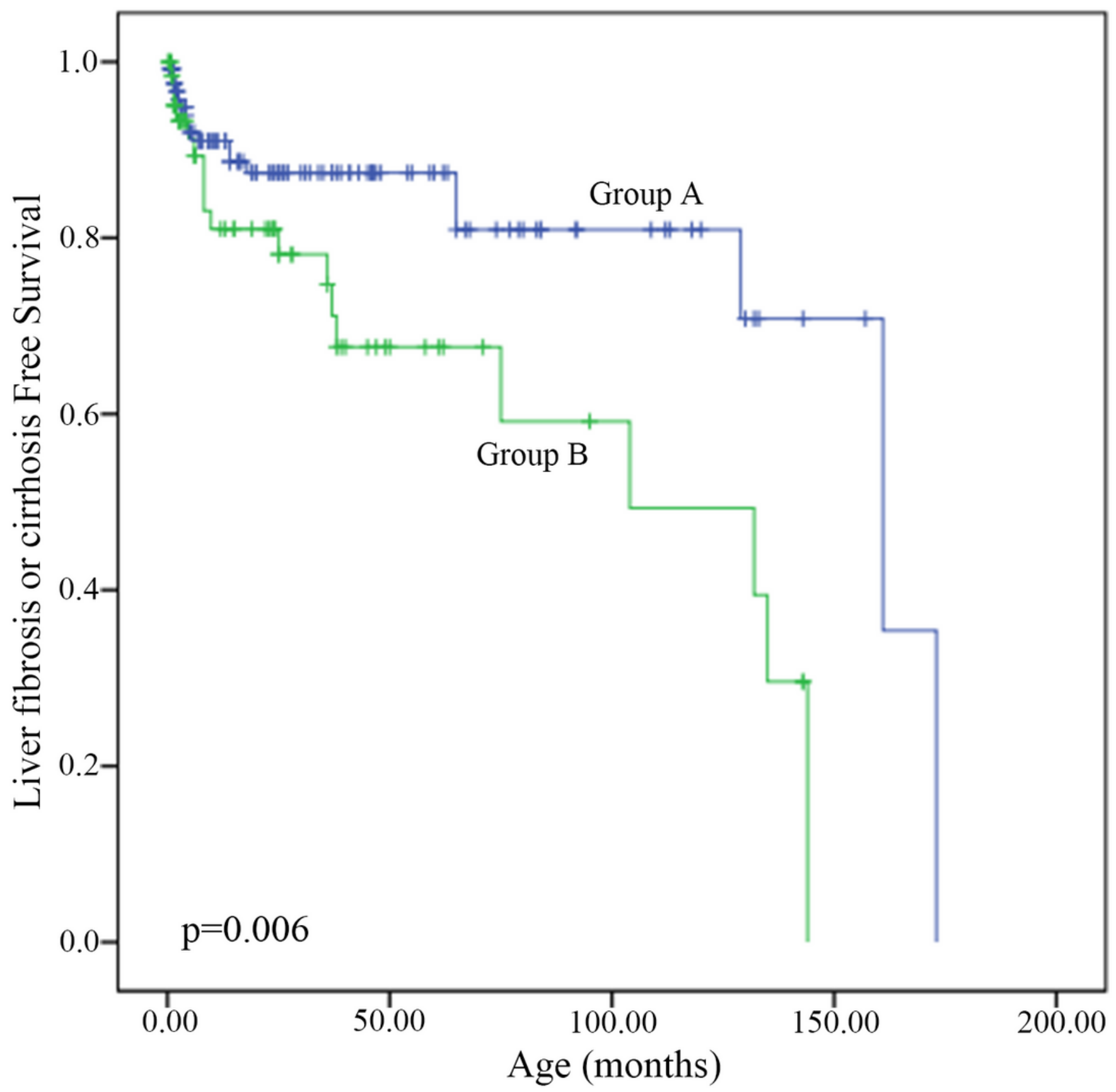

Figure 1

Prognostic analyses of CC patients with or without IHBD dilatation Kaplan-Meier analysis of liver fibrosis or cirrhosis free survival for group A (high) and group B (low) was analyzed. Log-rank test was used. 
a

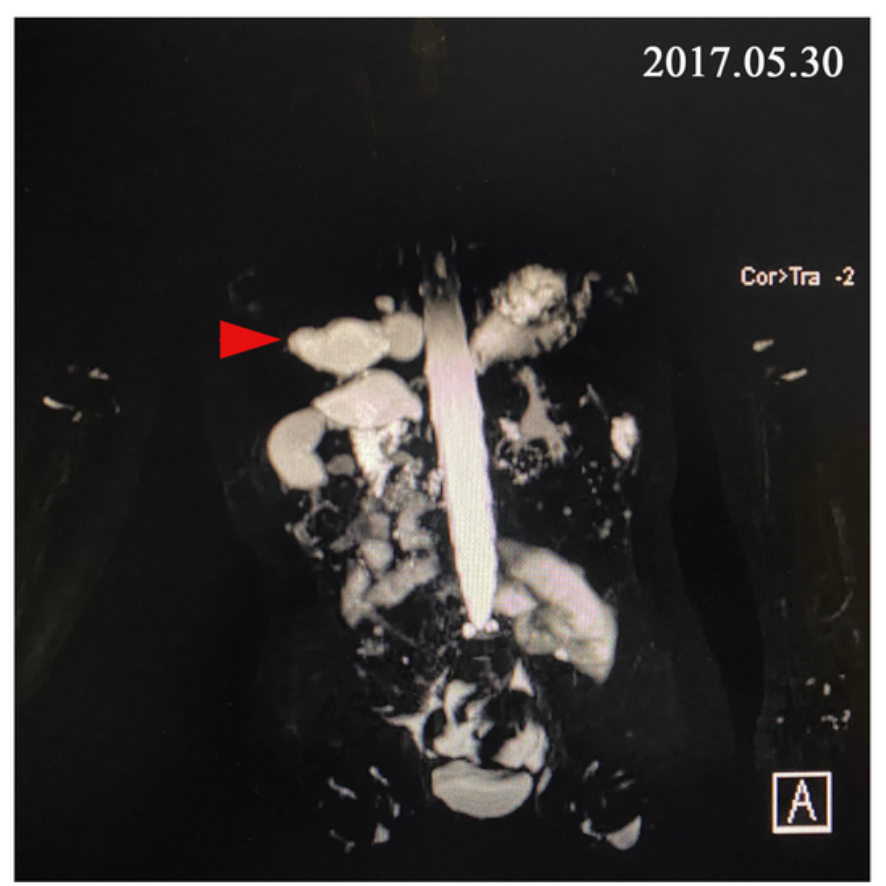

b

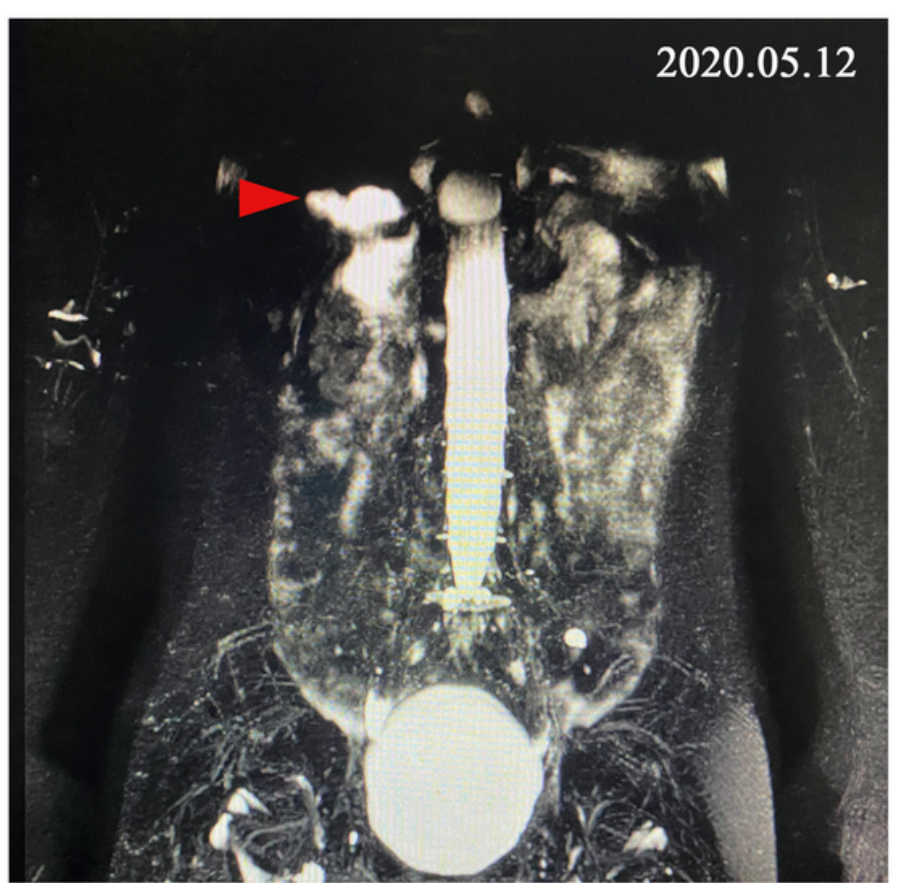

Figure 2

Pre- and postoperative MRCP images for one patient with IHBD The preoperative MRCP image (a) and postoperative MRCP image (b) were gained on May 30, 2017 and May 12, 2020, respectively. The red arrow represents the IHBD. 